\title{
LÓGICA DEÓNTICA, MUNDOS IDEALES Y MUNDOS PERFECTOS*
}

\author{
Jorge Luis Rodríguez \\ Universidad Nacional de Mar del Plata
}

RESUMEN. Las interpretaciones semánticas más fructíferas que se han ofrecido de los diversos sistemas axiomáticos de lógica deóntica se valen de la semántica de mundos posibles. Sin embargo, existen serios cuestionamientos contra dicha reconstrucción semántica en el dominio normativo. Entre aquellos de corte más filosófico se cuentan el fuerte compromiso ontológico que la semántica de mundos posibles requeriría asumir, así como la necesidad de aceptar que las normas serían susceptibles de verdad o falsedad. En el presente trabajo se intentará justificar que, no obstante tales objeciones, existe al menos un camino posible para preservar la riqueza de análisis que ofrece la semántica de mundos posibles para la lógica deóntica sin asumir tan problemáticos compromisos. Por otra parte, se examinarán algunas consecuencias significativas que se siguen de la utilización de esa interpretación semántica, centradas en la diferencia que media entre las nociones de mundos normativamente ideales y mundos normativamente perfectos.

Palabras clave: lógica, normas, semántica, mundos posibles.

\section{Deontic Logic, Ideal Worlds, and Perfect Worlds}

ABSTRACT. The most profitable semantic interpretations of the various axiomatic systems of deontic logic have been presented in terms of possible-worlds semantics. However, several objections have been raised against the use of this semantic framework in the normative domain. Among those most philosophical in character, it has been remarked the strong ontological commitment of possible-worlds semantics, as well as the need to assume in deontic logic that norms are capable of truth-values. In this paper I will try to maintain that, in spite of those objections, there is at least one possible way to preserve the analytical power of possible-worlds semantics for deontic logic without such problematic assumptions. Moreover, I will examine some interesting consequences that follow from this semantic interpretation, that stem from the difference between the notions of normatively ideal worlds and normatively perfect worlds.

Keywords: logic, norms, semantics, possible worlds.

\footnotetext{
* Fecha de recepción: 25 de junio de 2013. Fecha de aceptación: 20 de septiembre de 2013.
} 


\section{INTRODUCCIÓN}

unque en el diseño de un sistema lógico a veces prevalece una presenta-
ción sintáctica, en términos de axiomas y reglas de inferencia, y a veces
una presentación semántica, en términos de funciones de interpretación y
cláusulas para la atribución de verdad a las expresiones del lenguaje con-
siderado, lo cierto es que en general se estima que la actividad del lógico no se encuentra satisfactoriamente concluida si no se cuenta con ambas versiones, y se demuestra desde la metalógica que ambas presentaciones se corresponden una con la otra, en el sentido de que todo lo que es sintácticamente demostrable constituye una verdad lógica y todo lo que constituye una verdad lógica es sintácticamente demostrable (metateoremas de la representación, o de completitud y consistencia) ${ }^{1}$.

En el caso de la lógica deóntica, las interpretaciones semánticas más usuales y fructíferas que se han ofrecido de los diversos sistemas axiomáticos propuestos por los teóricos lo han sido en términos de la semántica de mundos posibles. El extraordinario desarrollo de la lógica modal alética a partir de su interpretación semántica en términos de mundos posibles tuvo impacto en el ámbito de la lógica deóntica sobre la base de las profundas analogías entre los conceptos aléticos y los deónticos.

Pese a ello, existen serios cuestionamientos contra la utilización de la semántica de mundos posibles como interpretación de los sistemas de lógica deóntica. En el presente trabajo examinaré en primer lugar — sin pretensión de exhaustividad, por ciertoalgunas de esas objeciones, concentrando la atención en aquellas más generales o de corte filosófico más que sobre las estrictamente técnicas. Intentaré justificar que, pese a ellas, es posible preservar la riqueza de análisis que ofrece la semántica de mundos posibles para el estudio de las relaciones lógicas entre las normas. En segundo lugar, trataré de mostrar algunas consecuencias interesantes que se siguen de la utilización de esa interpretación semántica, centradas en la diferencia entre las nociones de mundos normativamente ideales y mundos normativamente perfectos.

\section{LÓGICA DEÓNTICA, LÓGICA MODAL Y CUANTIFICACIÓN}

El surgimiento de la lógica deóntica estuvo profundamente vinculado con la puntualización de fuertes analogías entre los enunciados deónticos, los enunciados modales aléticos y los enunciados existenciales o cuantificados ${ }^{2}$. Esas analogías se verifican tanto en lo que respecta a las relaciones definicionales entre los conceptos fundamentales de estos tres grupos de enunciados, como en algunos principios lógicos básicos que parecen válidos en cada una de las tres áreas. Para mostrarlo de un modo simple, considérense las siguientes similitudes:

1 Vid. AlCHOURRÓN, 1995: 22-23; HAACK, 1978: 13-14.

2 Ello surge de la presentación del trabajo que ordinariamente se considera fundacional de la lógica deóntica, esto es, VON WRIGHT, 1951. No obstante, también VON WRIGHT se ha encargado de examinar detalladamente las diferencias que median, sobre todo, entre los conceptos modales aléticos y los deónticos, por ejemplo, en VON WRIGHT, 1983: 103 y ss. 
Enunciados existenciales:

$$
\Lambda x H x=d f \sim V x \sim H x
$$

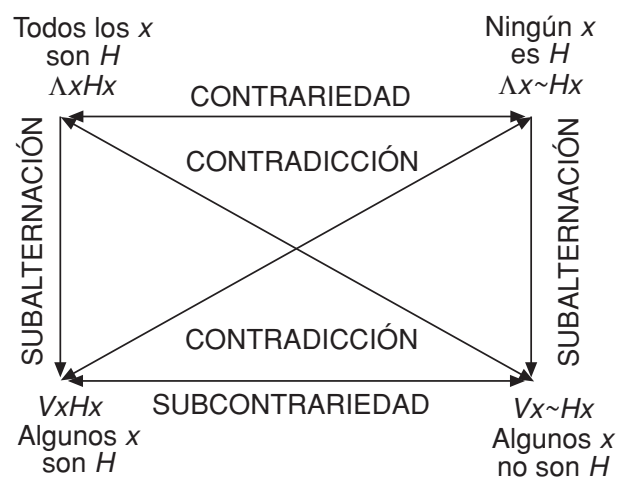

$(\Lambda x H x) \vee(\Lambda x \sim H x) \vee(V x H x \wedge V x \sim H x)$

\section{Enunciados modales aléticos:}

$$
N p=d f f \sim M \sim p
$$

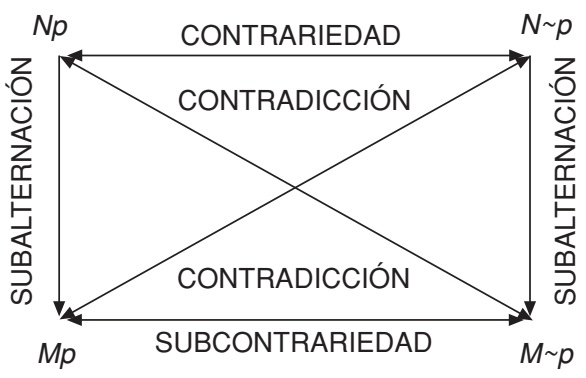

$(N p) \vee(N \sim p) \vee(M p \wedge M \sim p)$

\section{Enunciados deónticos:}

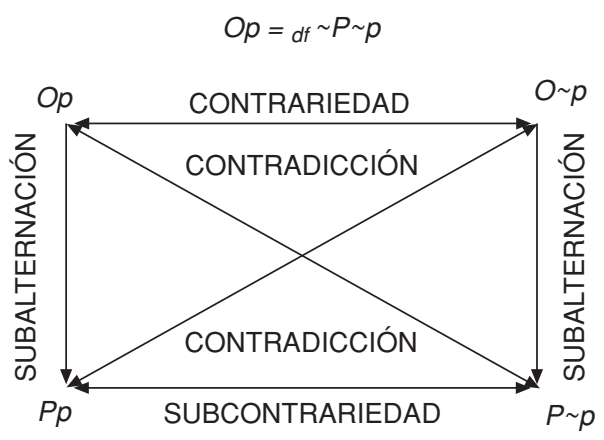

$(O p) \vee(O \sim p) \vee(P p \wedge P \sim p)$

Las indicadas analogías resultan tan fuertes que difícilmente puedan interpretarse como producto de una casualidad, de una suerte de «accidente filosófico». La mejor hipótesis explicativa parece consistir en que de las tres clases de enunciados, dos de ellas sean reducibles a la tercera. Y, por su carácter más básico, la mejor candidata para esa reducción es la clase de los enunciados existenciales o cuantificados. De hecho, existen muchos desarrollos teóricos que han propuesto reducir tanto los conceptos modales aléticos como los deónticos en términos de cuantificación sobre mundos posibles ${ }^{3}$.

3 Existen también propuestas de reducción de los enunciados deónticos directamente a enunciados modales aléticos ( $c f r$. KANGER, 1957; ANDERSON, 1958), pero esto no obsta a la tesis general que se sostiene en el texto si es que resulta a su vez posible reducir los enunciados modales aléticos en términos de enunciados existenciales. 
En lo que atañe a la lógica deóntica, en las décadas de los cincuenta y sesenta del siglo XX, y con el impulso de diferentes autores ${ }^{4}$, se desarrolló una interpretación semántica en términos de mundos posibles. Reseñaré aquí muy brevemente sólo la más conocida de tales presentaciones: el modelo kripkeano.

Un marco kripkeano es una estructura conformada por tres elementos $\left(M, m^{*}, R\right)$, donde $M$ es un conjunto no vacío de mundos posibles, $m^{*}$ es un elemento determinado de $M$ (el mundo real) y $R$ es una relación diádica de posibilidad relativa (relación de alternatividad o accesibilidad deóntica son las designaciones hoy más populares), que intuitivamente podría leerse del siguiente modo: un mundo $m$ que satisface dicha relación con el mundo real es tal que en él se cumple todo lo que es obligatorio en el mundo real. Un modelo kripkeano es un marco junto con una valuación $(v)$ que asigna valores 1 o 0 a las fórmulas de un lenguaje $L$ dado. La idea básica para el desarrollo de sistemas de lógica deóntica consiste en sostener las siguientes cláusulas de verdad para enunciados deónticos:

$$
\begin{aligned}
& v\left(O p, m^{*}\right)=1 \text { si } \Lambda m\left[R m^{*} m \rightarrow v(p, m)=1\right] \\
& v\left(P p, m^{*}\right)=1 \text { si } \operatorname{Vm}\left[R m^{*} m \wedge v(p, m)=1\right]
\end{aligned}
$$

Esto significa que la norma $O p$ es verdadera en el mundo real si y sólo si en todos los mundos deónticamente accesibles respecto del mundo real $p$ es verdadero, y la norma $P p$ es verdadera en el mundo real si y sólo si existe al menos un mundo normativamente accesible respecto del mundo real en el cual $p$ es verdadero. KRIPKE observó respecto de la lógica modal que las diversas propiedades formales de la relación $R$ se corresponden con diferentes sistemas lógicos. En el caso de la lógica deóntica, es posible igualmente obtener diferentes sistemas de acuerdo con qué propiedades se considere que satisface la relación $R$. Por ejemplo, si se toma en cuenta la clase de todos los marcos, esto es, si no se impone ninguna exigencia sobre la relación de accesibilidad deóntica, se obtiene un sistema débil de lógica deóntica, usualmente denominado $K D$ o $F$, caracterizado por el axioma:

$$
O(p \rightarrow q) \rightarrow(O p \rightarrow O q)
$$

Obsérvese que si en el mundo real valen $O(p \rightarrow q)$ y $O p$, entonces en todos los mundos normativamente accesibles respecto del mundo real sería verdadero tanto $(p \rightarrow q)$ como $p$, de lo cual se sigue por lógica proposicional que en todos los mundos normativamente accesibles sería también verdadero $q$. Por consiguiente, en el mundo real valdría igualmente $O q$.

Es posible, en cambio, tomar en consideración solamente la clase de todos los marcos en los que la relación $R$ es serial, esto es:

\section{$\Lambda m V m^{\prime}\left(m R m^{\prime}\right)$}

Esta exigencia sobre la relación $R$ determina que para todo mundo de $M$ existe al menos un mundo deónticamente accesible, y por referencia a esta clase de marcos, se obtiene el sistema estándar de lógica deóntica, caracterizado por el axioma:

$$
O p \rightarrow P p
$$

4 Fundamentalmente, Kanger, 1957; HintikKa, 1957; Montague, 1960, y KrIPKE, 1963. Para una excelente reseña del surgimiento de la semántica de mundos posibles para la lógica deóntica, vid. WOLEŃSKI, 1990. 
Ello así puesto que, supóngase que en el mundo real vale $O p$ pero, no obstante, no vale $P$. En tal caso, en todos los mundos normativamente accesibles respecto del mundo real es verdad que $p$, pero sin embargo no existe ningún mundo normativamente accesible en el cual sea verdadero $p$. Aunque pueda parecer lo contrario, estas dos afirmaciones son compatibles, dado que la afirmación de que para todo mundo posible, si éste es normativamente accesible respecto del mundo real, en él es verdadero $p$, es una afirmación general y condicional, que puede resultar verdadera vacuamente, esto es, si no existe ningún mundo normativamente accesible respecto del mundo real. Ahora bien, hemos asumido que la relación $R$ es serial, de modo que respecto de cualquier mundo debe existir al menos un mundo normativamente accesible. Pero si es verdad que en todos los mundos normativamente accesibles respecto del mundo real es verdad que $p$, y hay al menos un mundo normativamente accesible respecto del mundo real, en ese mundo ha de ser verdad que $p$, lo cual resultaría contradictorio con nuestra presuposición. En consecuencia, no puede existir ningún mundo en la clase de los marcos en los que la relación $R$ es serial en el que no valga que $O p \rightarrow P p$.

Como puede apreciarse, a partir de estos recursos relativamente simples y elegantes, la semántica de mundos posibles logra una gran riqueza de análisis, y un notable potencial teórico para dar cuenta de numerosos sistemas de lógica deóntica 5 .

\section{NORMAS, MUNDOS POSIBLES Y VERDAD}

Pese a lo señalado, se han dirigido muchas objeciones contra la semántica de mundos posibles para la lógica deóntica. Algunas de tales objeciones son de carácter general y filosófico, otras de carácter más técnico, específicamente lógicas. Entre las primeras se cuenta sin lugar a dudas en primer lugar el hecho de que la semántica de mundos posibles parece comprometernos con una ontología muy fuerte, casi de ciencia ficción: que existen, en el mismo sentido en el que existe nuestro mundo real, un sinnúmero de mundos posibles como realidades paralelas ${ }^{6}$.

Una primera línea de respuesta a este tipo de objeciones podría consistir en sostener que la lógica no necesita tomar partido sobre qué son los mundos posibles. De hecho, se han ofrecido las más diversas interpretaciones de dicha noción, y no todas ellas importan el mismo compromiso ontológico: objetos concretos, conjuntos de oraciones, funciones de interpretación del lenguaje objeto, proposiciones o conjuntos de ellas, estados de cosas, propiedades o conjuntos de ellas, entidades mentales o clases de ellas, maneras en las que las cosas podrían haber sido, ficciones, objetos no-existentes, construcciones teóricas, entre otras ${ }^{7}$.

Con todo, esta salida no parece enteramente convincente, y ello porque si bien la lógica no necesitará tomar partido, cualquiera que la utilice sí deberá necesariamente hacerlo, y en el caso de la semántica de mundos posibles en lógica deóntica, para

5 ÅQvisT, por ejemplo, desarrolla diez diferentes sistemas de lógica deóntica sobre la base de distintas propiedades de la relación de accesibilidad deóntica (cfr. ÅQVIST, 2002).

6 Así, por ejemplo, ALCHOURRÓN y MARTINO, 1988.

7 Sobre las distintas interpretaciones de la idea de mundos posibles y la defensa de este tipo de respuesta a la objeción considerada, vid. RöNNEDAL, 2010: 99-100. 
dar cuenta de un principio tan razonable como que obligatorio implica permitido es preciso asumir, como se dijo, que respecto de cualquier mundo existe al menos un mundo normativamente accesible, esto es, es preciso asumir un cierto compromiso ontológico.

Pero incluso aceptando esto último, habría que examinar cuán profundo es este compromiso ontológico. Para presentarlo de un modo simple, en la teoría de los mundos posibles existe una controversia no saldada en el modo de responder a un problema básico. Parecería que quien toma en serio la idea de mundos posibles ha de sostener, en primer lugar, que existe una pluralidad de mundos posibles (al menos más de uno); pero, en segundo lugar, también tiene que sostener que sólo uno de esos mundos es real, y que los restantes son meramente posibles. Ahora bien, las cosas meramente posibles no existen, de manera que, en aparente contradicción con la primera idea, sólo existiría un mundo posible.

La controversia se suscita entre dos posiciones básicas, que han sido denominadas posibilismo y actualismo. Ambas sostienen que el problema anterior deriva de una falacia de equívoco, pues las afirmaciones de que existen diversos mundos posibles y de que existe uno sólo que es real son ambas verdaderas en cierto sentido. No obstante, cada una de estas dos posiciones ubicaría el equívoco en un sitio diferente. Para los posibilistas, el equívoco se encuentra en el dominio de la cuantificación: la afirmación de que existen muchos mundos posibles resultaría verdadera si el dominio del cuantificador en ese enunciado es irrestricto. Sin embargo, muchas veces restringiríamos el dominio del cuantificador a subconjuntos propios de lo que existe (las cosas que realmente existen) y, en tal caso, el enunciado diría algo diferente ${ }^{8}$. Siguiendo la idea de QuiNE de que nuestra ontología depende de aquello que estemos dispuestos a reconocer como dominio de la cuantificación, desde este punto de vista todos los mundos posibles existen en el mismo sentido en el que existe el mundo real, sólo que el mundo real es nuestro mundo. Para el actualista, en cambio, lo que existe coincide con lo que es real, de modo que la cuantificación irrestricta equivale a la cuantificación sobre lo que es real. El equívoco se encuentra, para esta posición, en la clase de cosas a las que uno se refiere cuando emplea la expresión «mundo posible». Al decir que hay muchos mundos posibles nos referiríamos a posibles estados del mundo, a los diversos modos que podría asumir el mundo. Cuando se dice que sólo existe un mundo —el mundo real- estaríamos hablando de algo que se encuentra en uno de esos estados posibles. El mundo podría ser de muy diversos modos, y esos modos realmente existen, pero sólo uno de ellos se encuentra instanciado?.

Como puede apreciarse, el posibilismo $-\mathrm{o}$ realismo modal — asume un presupuesto ontológico muy fuerte: los mundos posibles existen en el mismo sentido en el que existe el mundo real. La «realidad» del mundo real dependería exclusivamente de una restricción en nuestro dominio de la cuantificación. El actualismo toma igualmente en serio la idea de los mundos posibles, pero no asume esta ontología recargada. Sólo existe el mundo real; los «mundos posibles» alternativos al mundo real son simplemente construcciones teóricas, representaciones concebibles acerca de cómo podría ser el

8 Vid., por ejemplo, LEWIS, 1986: 1-50.

9 Vid. Stalnaker, 2003: 6-7. 
mundo si fuese distinto de cómo es. Desde este punto de vista, por ejemplo, decir que $p$ es necesariamente verdadero en el mundo real depende de que imaginemos cómo podría ser el mundo real más allá de cómo es, y de que en todas esas representaciones concebibles $p$ sea verdadero, lo que en modo alguno supone atribuirle existencia a otros mundos. Algo semejante podría sostenerse respecto de los conceptos deónticos: decir que $p$ es obligatorio en el mundo real dependería de que imaginemos cómo podría ser el mundo si fuera normativamente mejor de lo que en verdad es, y que $p$ sea verdadero en todas esas representaciones concebibles que estimamos normativamente accesibles.

Suponiendo que pudiese aceptarse esta interpretación ontológicamente débil de la idea de mundos posibles como respuesta a la primera objeción considerada, de todos modos subsiste una segunda y muy seria objeción de corte filosófico contra la aplicación de la semántica de mundos posibles para el análisis de la lógica deóntica: ella parece comprometernos con la asunción de que las normas son susceptibles de valores de verdad, lo cual resulta contraintuitivo ${ }^{10}$. Tal como lo presentara JøRGENSEN en términos de un dilema, bajo el supuesto de que las normas no son ni verdaderas ni falsas, o bien la lógica circunscribe su alcance a entidades susceptibles de verdad o falsedad, en cuyo caso no es posible una lógica de normas, o bien una lógica de normas es posible, pero entonces el dominio de la lógica es más amplio que el de la verdad ${ }^{11}$. Simplificando un panorama un poco más complejo, podría decirse que si no estamos dispuestos a aceptar el primer cuerno del dilema, esto es, que no es posible una lógica de normas, debido a que ello parece contradecir intuiciones muy fuertes acerca del modo en el que argumentamos a partir de normas, las alternativas para la superación del dilema parecerían reducirse a dos: o bien afrontamos su segundo cuerno, es decir, contrariando una muy fuerte tradición en lógica, justificamos que la lógica tiene un alcance más amplio que la verdad, o bien rechazamos el presupuesto del cual parte el dilema y aceptamos que, después de todo, las normas son susceptibles de valores de verdad.

En trabajos anteriores me he inclinado por la segunda de estas alternativas ${ }^{12}$, sobre la base de uno de los intentos más interesantes que se han ensayado desde este punto de vista que es, a mi juicio, el desarrollado por ALCHOURRÓN y MARTINO ${ }^{13}$. Allí se propone que tanto la noción sintáctica como la noción semántica de consecuencia lógica presentarían ciertos rasgos comunes, que podrían recogerse en una noción abstracta de consecuencia, a partir de la cual sería posible caracterizar las nociones lógicas fundamentales, como la de contradicción o las conectivas lógicas, sin apelar a las nociones

10 Cabría, por supuesto, preguntarse con qué concepción o concepciones de la verdad parece comprometernos la semántica de mundos posibles y bajo qué concepción o concepciones de la verdad resulta contraintuitivo atribuir valores de verdad a las normas. Sin poder extenderme adecuadamente sobre estas complejas cuestiones, sólo diré con relación a la primera pregunta que si bien las cláusulas de verdad para las normas en la semántica ordinaria de mundos posibles parece asumir alguna versión de la teoría correspondentista, en realidad no resultaría incompatible con concepciones coherentistas, pragmatistas, minimalistas o deflacionistas (cfr. RÖNNEDAL, 2010: 107-108). Con relación a la segunda pregunta, sólo puedo decir que, salvo demostración en contrario, las normas no parecen entidades semejantes a las proposiciones, esto es, susceptibles de valores de verdad bajo ninguna concepción de la verdad.

11 Cfr. JøRGENSEN, 1937-1938.

12 Por ejemplo, FERrER BELtrÁn y RodrígueZ, 2011: 40-46.

13 Cfr. Alchourrón y Martino, 1988. 
de verdad y falsedad, posibilitando de tal suerte extender el campo de aplicación de la lógica a dominios que exceden el de la verdad, como sería el caso de la lógica deóntica. Lamentablemente, la sugerencia concreta de cómo desarrollar sobre tales bases un sistema de lógica deóntica que postulan ALCHOURRÓN y MARTINO sobre el final de su trabajo presenta serias dificultades técnicas, tal como lo ha puesto de manifiesto con claridad H. ZULETA ${ }^{14}$. Y si bien las críticas de ZULETA no mellan la propuesta general de los autores, lo cierto es que no existe hoy un desarrollo satisfactorio de una lógica deóntica desde esta perspectiva.

La aplicación al campo de la lógica deóntica de la semántica de mundos posibles parece en cambio comprometernos con la salida alternativa para la superación del dilema de JØRGENSEN, esto es, la admisión de que las normas son susceptibles de verdad o falsedad. Como bien lo señala D. MAKINSON, considerar que las normas no son susceptibles de verdad o falsedad pero que, no obstante, a los fines de la lógica pueden ser tratadas como si los tuvieran, tal como ocurre en la mayor parte de los casos cuando se emplea en este dominio la semántica de mundos posibles, al menos sin alguna explicación adicional resulta una posición «...carente de todo principio y próxima a la inconsistencia...» ${ }^{15}$.

No obstante, se han ensayado diferentes propuestas tratando de aprovechar los recursos de la semántica de mundos posibles para la lógica deóntica pero preservando la intuición de que las normas en sí mismas no son susceptibles de verdad o falsedad. Una de las más conocidas de estas propuestas consiste en el uso de la semántica de mundos posibles para el desarrollo de una lógica para enunciados normativos interpretados descriptivamente, esto es, como proposiciones normativas ${ }^{16}$. El modo más simple de hacer esto consiste en asumir que los enunciados normativos como $O p$, interpretados descriptivamente, esto es, como proposiciones relativas al contenido de un cierto sistema normativo, reflejan las propiedades lógicas de las normas, con lo cual una lógica de proposiciones normativas nos ofrecería un modo indirecto de examinar el comportamiento lógico de las normas ${ }^{17}$.

El problema con este enfoque es que, como ALCHOURRÓN se encargara de demos$\operatorname{trar}^{18}$, una lógica de proposiciones normativas no se comporta igual que una genuina lógica de normas, salvo en el supuesto de completitud y consistencia del sistema normativo de referencia. En consecuencia, el punto de partida de este enfoque resulta erróneo.

14 Cfr. ZuletA, 2008: 66-70.

15 Cfr. MaKinson, 1999: 30.

16 Sobre la distinción entre normas y proposiciones normativas, vid. VON WRIGHT, 1963 a: 106.

17 Este punto de vista fue el sostenido en VON WRIGHT, 1963a, si bien VON WRIGHT no adopta la semántica de mundos posibles. Es curioso que la misma idea sea todavía defendida por algunos autores pese a la demostración de AlCHOURRÓn, 1969, que se comenta a continuación en el texto. Por ejemplo, S. HANSSON (2006: 334-335) sostiene que: «No es entonces... problemático utilizar una lógica veritativo-funcional para ofrecer un modelo de (una materia expresada por) oraciones que no son verdaderas o falsas... Existe una correspondencia uno a uno entre oraciones del tipo «debo pagar esta deuda» y oraciones del tipo «hay una norma válida según la cual debo pagar esta deuda». Las oraciones del segundo tipo pueden ser verdaderas o falsas. En la medida en que un sistema lógico refleje adecuadamente las propiedades de las oraciones del segundo tipo, también reflejará, indirectamente, a las del primer tipo».

18 Cfr. Alchourrón, 1969. 
Se podría, por supuesto, abandonar la pretendida isomorfía entre lógica de normas y lógica de proposiciones normativas, aceptar la imposibilidad de una genuina lógica de normas, y releer la lógica deóntica como una lógica para proposiciones normativas relativas a un sistema normativo dado ${ }^{19}$. Pero este camino, aunque cuenta con contribuciones importantes, tampoco está libre de dificultades. El punto crucial sigue derivándose de la demostración de AlCHOURRón antes comentada. Porque lo que demuestra el trabajo de AlCHOURRÓN es que los principios lógicos que parecen caracterizar a una genuina lógica de normas, como que si una conducta es obligatoria no puede al propio tiempo estar prohibida, o que una conducta o bien es obligatoria, o bien está prohibida, o bien es facultativa, no valen sin más para una lógica de proposiciones relativas a un cierto sistema normativo, incluso asumiendo que este último no se limita a contener ciertas normas explícitamente promulgadas sino que comprende todas las consecuencias lógicas que de ellas se siguen. En otras palabras, ALCHOURRÓn no demuestra sino que asume que una lógica de normas es posible, y su prueba posee la siguiente estructura condicional: si es posible una lógica de normas y si los sistemas normativos se interpretan como comprensivos de todas las consecuencias que es posible derivar de acuerdo con los principios de esa lógica de normas a partir de ciertas normas explícitamente promulgadas, aun así la lógica de proposiciones normativas no equivale a la lógica de normas sino bajo ciertos supuestos excepcionales.

Siendo ello así, si se asume que las normas no son susceptibles de verdad o falsedad, se abandona por ello la pretensión de desarrollar una genuina lógica de normas, y se interpreta a la lógica deóntica como una lógica de proposiciones descriptivas del contenido de un cierto sistema normativo, surge inmediatamente la pregunta de qué relevancia podría tener un cálculo semejante si el sistema normativo de referencia no contiene todas sus consecuencias lógicas ${ }^{20}$. Para decirlo de otro modo, parece que nos enfrentamos aquí al siguiente dilema: o bien consideramos que cada sistema normativo está exclusivamente conformado por las normas que han sido dictadas por ciertas autoridades, en cuyo caso para una descripción adecuada de un sistema semejante no se requerirá otra cosa que lógica proposicional y de predicados, de modo que no existiría ninguna ley específica de una supuesta lógica de proposiciones normativas, o bien hay leyes específicas de la lógica de proposiciones normativas, pero entonces ha de aceptarse que los sistemas normativos no se limitan a contener ciertas normas expresamente dictadas por ciertas autoridades, sino además las consecuencias lógicas que se sigan de ellas de acuerdo con algún sistema de lógica de normas ${ }^{21}$. En síntesis, o bien la lógica deóntica interpretada como una lógica de proposiciones normativas presupone una genuina lógica de normas, o bien no es más que el cálculo lógico

19 Este es el punto de vista que se propone en ALCHOURRÓN y BulYGin, 1981, y parece seguirse en ALCHOURRÓN, 1993.

${ }^{20}$ En VON WRIGHT, 2000, se propone un sistema de lógica para proposiciones normativas en el cual no se presupone que los sistemas normativos de referencia contengan todas sus consecuencias lógicas.

${ }_{21}$ Cfr. Rodríguez, 2003; en el mismo sentido, ZuLETA, 2008: 57. Los sistemas desarrollados en MAKINSON, 1999, y MAKINSON y VAN DER TORRE, 2000, 2001 y 2003, no escapan a esta objeción: en ellos el sistema normativo de referencia se toma como una caja negra que recibe como inputs informaciones sobre circunstancias fácticas y permite derivar como outputs consecuencias normativas. Pero en las diferentes modelizaciones de los outputs se toman en consideración todas las consecuencias que se siguen de las circunstancias fácticas y las normas del sistema, lo cual supone que implícitamente se están aceptando relaciones lógicas entre las normas. 
ordinario aplicado a la descripción de los actos de promulgación y derogación de normas.

Existe, con todo, una posibilidad de utilizar la semántica de mundos posibles para construir una genuina lógica de normas, no de proposiciones que se refieren a ellas, preservando la idea de que las normas no son susceptibles de verdad o falsedad. Se trata de una alternativa de superación del dilema de JøRGENSEN que asume su segundo cuerno, esto es, que el alcance de la lógica es más amplio que el de la verdad, como en la propuesta de ALCHOURRÓn y MARTINO, conservando el potencial de la semántica de mundos posibles. La idea consistiría en sostener que la diferencia entre decir que $p$ es el caso y decir que $p$ debe ser el caso se localiza en el uso del lenguaje, en la diferente actitud proposicional adoptada en cada situación. Cuando el lenguaje se emplea descriptivamente se pretende con él representar fielmente la realidad. Si se refleja correctamente lo que acontece, el enunciado formulado será verdadero; en caso contrario será falso y correspondería corregirlo para adecuarlo a la finalidad perseguida. Apelando a la conocida distinción de ANSCOMBE ${ }^{22}$, podríamos decir que en este caso la dirección de ajuste va del lenguaje al mundo, el lenguaje trata de ajustarse al mundo, de modo que en caso de discordancia el problema está en todo caso en el lenguaje. En cambio, cuando el lenguaje se emplea prescriptivamente, se pretende con él influir sobre la conducta de cierto agente. Si la conducta del destinatario de la prescripción no se ajusta a lo prescripto, no hay razón alguna para corregir la prescripción: lo que en todo caso debería corregirse es la conducta para que ella se adecue a lo prescripto. La dirección de ajuste en este caso es inversa: va del mundo al lenguaje, en el sentido de que en caso de discordancia el problema no está en el lenguaje sino en el mundo.

A diferencia de la utilización estándar de la semántica de mundos posibles para el análisis de las normas, en la que se interpreta que las normas son verdaderas si describen adecuadamente lo que acontece en ciertos mundos normativamente accesibles, podríamos decir que las normas son supuestos de uso prescriptivo del lenguaje, donde la dirección de ajuste resultaría del mundo al lenguaje. Desde este punto de vista, prescribir que es obligatorio $p$ en el mundo real significaría preferir como mundos normativamente accesibles a su respecto mundos en los que es verdad que $p$. La enunciación de una norma importaría establecer una preferencia, una selección de ciertos mundos como normativamente accesibles, no una descripción de lo que en ellos acontece, y a partir de esta idea sería posible igualmente elaborar una semántica para la lógica deóntica en términos de mundos posibles.

Es fácil advertir que, así como se verifican fuertes analogías entre los conceptos deónticos y los aléticos, también existen fuertes analogías entre los conceptos deónticos y las preferencias. Tomando como base los conceptos de preferencia fuerte [«...es mejor que...» (>)] y preferencia débil [《...es al menos tan bueno como...» $(\geq)$ ], y sin pretensión de reducir las normas a simples preferencias, en un trabajo anterior he señalado algunas de tales analogías ${ }^{23}$, por ejemplo:

22 Cfr. AnsCOMBE, 1957: 56

23 Cfr. RodrígueZ, 2002: 211-240. Vid. igualmente, vON WRIGHT, 1963 b. 
Normas y preferencias:
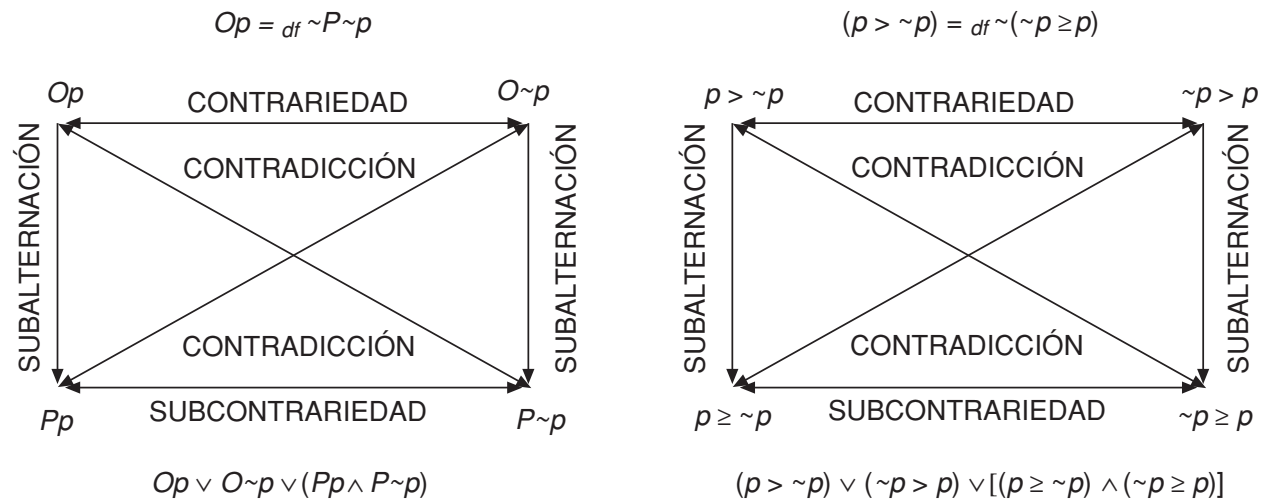

Es importante advertir que existe un aspecto en que la similitud entre los conceptos deónticos y las preferencias es todavía mayor que la que media entre los conceptos deónticos, los existenciales y los aléticos, puesto que mientras para estos últimos valen:

$$
\begin{aligned}
\Lambda x H x & \rightarrow H a \rightarrow \mathrm{V} x H x \\
N p & \rightarrow p \rightarrow M p
\end{aligned}
$$

sus análogos en lógica deóntica y lógica de preferencias no valen:

$$
\begin{array}{ll}
\leftarrow O p \rightarrow p & \leftarrow(p>\sim p) \rightarrow p \\
\leftarrow p \rightarrow P p & \leftarrow p \rightarrow(p \geq \sim p)
\end{array}
$$

Por supuesto, las normas no pueden asimilarse tan sencillamente a preferencias. No obstante, en distintos trabajos S. HANSSON ha desarrollado sistemas de lógica deóntica que pueden interpretarse como inscriptos en esta línea ${ }^{24}$. HANSSON diseña una lógica deóntica basada en la lógica de preferencias de conformidad con el principio de que los predicados de obligación son contranegativos respecto de la relación de preferencia débil ( $\geq$-contranegativos). Para explicarlo brevemente, en lugar de asimilar de manera directa un enunciado como $\mathrm{O} p$ como una preferencia de los mundos $p$ sobre los mundos $\sim p$, tal como parece sugerirse en las analogías anteriores, HANSSON considera que el predicado obligatorio satisface la siguiente propiedad: para todo $p$ y todo $q$,

$$
[O p \wedge(\sim p \geq \sim q)] \rightarrow O q .
$$

No es mi intención aquí examinar la plausibilidad de tales sistemas, sino solamente resaltar que a través de estrategias como éstas es posible preservar la elegancia y riqueza de análisis que ofrece la semántica de mundos posibles para la lógica deóntica sin comprometerse con la problemática tesis de que las normas son susceptibles de verdad o falsedad. 


\section{MUNDOS NORMATIVAMENTE IDEALES Y MUNDOS NORMATIVAMENTE PERFECTOS}

Sea $m \in M$ tal que $m^{*} R m$, esto es, que $m$ satisface la relación de accesibilidad deóntica respecto de $m^{*}$ (es una alternativa deóntica de $m^{*}$, o es deónticamente permisible respecto de $\left.m^{*}\right)$. Como señalé, la idea que pretende capturar esta relación es que $m$ es, en tal caso, un mundo en el cual se verifica todo lo que es obligatorio en $m^{*}$. En razón de ello podríamos decir que $m$ constituye un mundo normativamente ideal respecto de $m^{*}$. De acuerdo con esto, ser un mundo normativamente ideal es una noción relativa (a cierto mundo, no necesariamente distinto de sí mismo).

Es preciso reconocer que incluso quienes aceptan una semántica de mundos posibles para la lógica deóntica han dirigido objeciones contra esta interpretación en términos de mundos normativamente ideales. En primer lugar se podría objetar que aunque tengamos una cierta ordenación de mundos posibles, esa ordenación podría no resultar suficiente como para hablar de mundos ideales, pues podría acontecer que la ordenación en cuestión, aunque esté determinada por una relación de preferencia transitiva y completa, no garantice que haya mundos que sean mejores que todos los demás. Sin embargo, cuando se apela a la idea de mundos ideales en el contexto de una semántica para la lógica deóntica, a lo que se alude es a una noción de «idealidad normativa», en el sentido de que las obligaciones se cumplan, no necesariamente en un sentido más general que permita decir que un mundo es, todas las cosas consideradas, mejor que otro ${ }^{25}$.

Ahora bien, en segundo lugar, se ha observado que en un mundo normativamente ideal, como nadie haría nada incorrecto, no habría ningún deber de actuar para evitar actos incorrectos ni para compensar por la realización de actos incorrectos. La apelación a mundos normativamente ideales tendría, pues, consecuencias contraintuitivas para la lógica deóntica, que han sido recogidas en algunas muy conocidas paradojas, entre ellas:

$$
O \sim p \rightarrow O(p \rightarrow q) \quad \text { Paradoja de las obligaciones derivadas }{ }^{26}
$$

Esta fórmula, válida en el sistema estándar de lógica deóntica, admite interpretaciones como «si está prohibido matar, entonces es obligatorio que si se mata también se robe», lo cual no parece sensato. Lo mismo ocurre con otra expresión proposicionalmente equivalente a la anterior, como:

$$
O p \rightarrow O(p \vee q) \quad \text { Paradoja de Ross }{ }^{27}
$$

que podría leerse como «si es obligatorio despachar una carta, es obligatorio despacharla o quemarla».

Todo esto sería consecuencia de que para determinar lo que se debe hacer deberían considerarse no sólo mundos ideales sino también mundos subideales. La referencia a mundos normativamente ideales nos recomendaría actuar como si realmente

\footnotetext{
25 Cfr. Hansson, 2006: 331.

26 Cfr. PRIOR, 1954.

27 Cfr. Ross, 1941.
} 
viviéramos en un mundo semejante, lo que no siempre sería una recomendación adecuada $^{28}$.

Aunque no es posible en el marco de este trabajo considerar en toda su extensión paradojas como las comentadas, es preciso reconocer que, efectivamente, el sistema estándar de lógica deóntica, con su interpretación semántica en términos de mundos normativamente ideales, no permite dar cuenta adecuadamente, por ejemplo, de las normas condicionales, ni tampoco de nuestras obligaciones en circunstancias subideales: por referencia a mundos normativamente ideales no es posible explicar adecuadamente que podemos tener ciertas obligaciones derivadas del incumplimiento de otras obligaciones. De todos modos, esto todavía no es suficiente para descalificar la apelación a mundos normativamente ideales: es posible salvar estas objeciones pensando que la apelación a mundos normativamente ideales permite dar cuenta de al menos un primer nivel de intuiciones sobre las relaciones lógicas entre normas (las relaciones entre los diferentes operadores deónticos, la idea de que obligatorio implica permitido, la distributividad de los operadores deónticos por la conjunción y la disyunción, etc.). Pero además, la necesidad de considerar mundos subideales en realidad no descalifica sino que presupone la consideración de mundos normativamente ideales: lo que en todo caso exigiría es una complejización de la reconstrucción, no una sustitución de la idea de mundos normativamente ideales.

Es, creo, muy importante diferenciar la idea de un mundo normativamente ideal, en el sentido antes considerado, de lo que podría denominarse un mundo normativamente perfecto. J. HiNTIKKA ha efectuado una comparación muy interesante de los mundos normativamente ideales con la idea kantiana del reino de los fines. La noción de una alternativa deóntica o mundo normativamente ideal puede considerarse una variante más débil y relativizada de la noción kantiana de un reino de los fines: más débil porque no hace referencia a ningún principio moral particular, como el imperativo categórico kantiano o cualquier otro; relativizada porque la noción de una alternativa deóntica o mundo normativamente ideal es relativa a un cierto mundo que se toma como punto de referencia, tal como ya se indicó. Por otra parte, mientras el reino de los fines kantiano parece ser concebido como único, ordinariamente se acepta que puede haber diversos mundos normativamente ideales respecto de un cierto mundo. Pero lo más interesante de esta comparación es que HiNTIKKA sugiere que si se interpreta la idea del reino de los fines kantiano como una alternativa deóntica al mundo real, esa alternativa debería concebirse como un mundo deónticamente perfecto, esto es, un mundo en el que todas las obligaciones, no solo las que valen en el mundo real sino también aquellas que valen para ese mundo alternativo, se satisfacen ${ }^{29}$. Podríamos entonces definir a un mundo normativamente perfecto en los siguientes términos:

$m \in M$ es normativamente perfecto si $m R m$, esto es, si en él vale $O p \rightarrow p$.

En otras palabras, un mundo sería normativamente perfecto si es normativamente ideal respecto de sí mismo, esto es, si la relación de accesibilidad deóntica fuese reflexiva, validando así el principio $O p \rightarrow p$. Ahora bien, la validez de este principio no es algo que pueda aceptarse en general para una lógica deóntica, de modo que pensar en

28 Cfr. Hansson, 2006: 332-333.

29 Cfr. HintikKA, 1971: 73. 
un sistema de lógica deóntica basado en la clase de los marcos en los que la relación $R$ es reflexiva parece inadecuado. Sin embargo, existe una alternativa más débil, que se corresponde con la idea sugerida por HINTIKKA: un sistema de lógica deóntica basado en la clase de marcos en los que la relación $R$ es secundariamente reflexiva:

$$
\Lambda m^{\prime}\left(m R m^{\prime} \rightarrow m^{\prime} R m^{\prime}\right)
$$

En otras palabras, que si un mundo es normativamente ideal respecto de otro entonces es deónticamente ideal respecto de sí mismo, esto es, es un mundo normativamente perfecto. El sistema resultante, denominado usualmente $D M$ o $d T^{\prime}$, es una extensión del sistema estándar de lógica deóntica en el que vale el siguiente axioma:

$$
O(O p \rightarrow p)
$$

Supóngase que $O(O p \rightarrow p)$ no fuera válido en algún mundo $m$ en un modelo basado en un marco secundariamente reflexivo. Debería haber un mundo $m$ ' deónticamente ideal respecto de $m$ en el cual $O p \rightarrow p$ fuese falso, de modo que $O p$ sería verdadero y $p$ falso en $m$ '. Pero como el marco es secundariamente reflexivo, se cumple que $m$ 'R $m$ ', por lo cual vale en $m^{\prime}$ que $O p \rightarrow p$, lo cual es contradictorio. En consecuencia, no puede haber ningún mundo basado en un marco secundariamente reflexivo en el cual $O(O p \rightarrow p)$ sea falso ${ }^{30}$.

$O(O p \rightarrow p)$ es un principio muy discutido en lógica deóntica puesto que es uno de los pocos principios que podrían ser admitidos en su seno que tiene la estructura $O A$, donde $A$ no es algo trivial, y parece un principio sensato para la lógica deóntica si tomamos como parámetro de referencia normativa mundos normativamente perfectos, esto es, si restringimos la consideración de los mundos normativamente ideales a aquellos que resultan normativamente perfectos en el sentido de que todas las obligaciones se cumplen y ninguna prohibición se transgrede. De hecho, resulta difícil entender en qué sentido se podría considerar a un mundo como normativamente ideal respecto del nuestro si en él no se satisface todo lo que resulta obligatorio, no sólo en nuestro mundo sino también en él mismo, pues en tal caso estaríamos tomando como estándar para la evaluación de las relaciones lógicas entre normas mundos en los que todas sus obligaciones podrían ser incumplidas y sus prohibiciones transgredidas.

Con todo, existe una consecuencia que debería tenerse bien presente si se asume este punto de vista para el diseño de un sistema de lógica deóntica. En nuestro mundo - el real — no todas las obligaciones se cumplen ni todas las prohibiciones se respetan, de manera tal que nuestro mundo está lejos de ser un mundo normativamente perfecto. Pero entonces, tal como lo sostiene CHELLAS, si hay al menos una acción $p$ tal que $p$ no es el caso en nuestro mundo pese a ser obligatoria, nuestro mundo no podría ser normativamente ideal respecto de ningún otro mundo, ya que hemos asumido que en todos los mundos normativamente ideales son normativamente perfectos, esto es, que en ellos vale que $O p \rightarrow p^{31}$.

Lo expuesto tiene diferentes consecuencias según cuál sea la interpretación que se asigne a la semántica de mundos posibles para la lógica deóntica. Si asumimos que las normas tienen una dirección de ajuste del mundo al lenguaje, la cuestión no parece

30 Cfr. RönNEDAL, 2010: 132.

31 Cfr. Chellas, 1980: 193-194. 
problemática dado que en tal caso son las propias normas las que seleccionan a ciertos mundos como normativamente ideales, esto es, las que determinan el codominio de la relación de accesibilidad normativa. Si en cambio se asume que las normas poseen una dirección de ajuste del lenguaje al mundo, y se concibe a los mundos posibles desde la perspectiva del actualismo, esto es, la interpretación ontológicamente débil de la idea de mundos posibles, tampoco parece demasiado problemática la observación señalada, puesto que se limitaría a consignar que los modos de ser alternativos que podemos concebir de nuestro mundo, a los fines de la reflexión deóntica, son siempre alternativas concebibles mejores de lo que el mundo efectivamente es. Pero quien asuma en cambio que las normas son verdaderas o falsas por referencia a mundos posibles concebidos desde la perspectiva del posibilismo, esto es, la interpretación ontológicamente cargada de los mundos posibles, parece forzado a optar por una de estas dos alternativas: o bien ha de renunciar a evaluar la lógica deóntica por apelación a mundos normativamente perfectos, renunciando así a tomar como axioma $O(O p \rightarrow p)$, o bien ha de aceptar que, como el mundo real no podría ser normativamente ideal respecto de ningún otro mundo, vivimos en uno de los peores mundos, en el sentido de que ningún mundo sería normativamente peor que el nuestro. LEIBNIZ decía que el universo, esto es, el mundo real, era uno de un número infinito de mundos posibles existentes en la mente de Dios. Valiéndonos de esta terminología religiosa podríamos decir que el posibilismo, o bien debe renunciar al Paraíso, o bien ha de reconocer que estamos condenados a vivir en el Infierno.

\section{REFERENCIAS}

AlChOURRÓN, C. E., 1969: «Logic of Norms and Logic of Normative Propositions», Logique et Analyse, 12, 47: 242-268.

- 1993: «Philosophical Foundations of Deontic Logic and the Logic of Defeasible Conditionals», en MeYER y WIERINGA, 1993: 43-84.

- 1995: «Concepciones de la lógica», en Alchourrón, MÉNDEZ y OrAYEn, 1995: 11-48.

Alchourrón, C. E., y BulYgin, E., 1981: «The Expressive Conception of Norms», en HiLPINEN, 1981: 95-124.

AlChOURRón, C. E., y MARTinO, A., 1988: «Lógica sin verdad», Teoría, segunda época, año 3, 1987-1988, núms. 7-8: 7-43.

Alchourrón, C. E.; MéNDEZ, J., y ORAYEN, R. (eds.), 1995: Enciclopedia Iberoamericana de Filosofía, vol. 7, Lógica, Madrid: Trotta-CSIC.

Anderson, A. R., 1958: «A Reduction of Deontic Logic to Alethic Modal Logic», Mind, 67, núm. 265: 100-103.

Anscombe, G. E. M., 1957: Intention, Oxford: Basil Blackwell.

ÅQvisT, L., 2002: «Deontic Logic», en GABBAY y GUENTHNER, 2002: 147-264.

Chellas, B. F., 1980: Modal Logic. An Introduction, Cambridge: Cambridge University Press.

FERRER Beltrán, J., y RODRíGUEZ, J. L., 2011: Jerarquías normativas y dinámica de los sistemas jurídicos, Madrid-Barcelona-Buenos Aires: Marcial Pons.

Gabbay, D., y Guenthner, F. (eds.), 2002: Handbook of Pbilosophical Logic, vol. 8, 2." ed., Dordrecht: Kluwer Academic Publishers.

HAACK, S., 1978: Philosophy of Logics, Cambridge: Cambridge University Press. 
Hansson, S. O., 2001: The Structure of Values and Norms, Cambridge: Cambridge University Press.

- 2004: «A New Representation Theorem for Contranegative Deontic Logic», Studia Logica, 77: $1-7$.

— 2006: «Ideal Worlds - Wishful Thinking in Deontic Logic», Studia Logica, 82: 329.

Hilpinen, R. (ed.), 1971: Deontic Logic: Introductory and Systematic Readings, Dordrecht-Boston-London: Reidel.

- 1981: New Studies in Deontic Logic, Dordrecht-Boston-London: Reidel.

HintikKA, J., 1957: «Quantifiers in Deontic Logic», Societas Scientiarum Fennica, Commentationes Humanarum Literarum, XXIII, 4.

— 1971: «Some Main Problems in Deontic logic», en HilPinen, 1971: 59-104.

JørGENSEN, J., 1937-1938: «Imperatives and Logic», Erkenntnis, t. 7: 288-296.

KangeR, S., 1957: «New Foundations for Ethical Theory», Stockholm: Almqvist \& Wiksell, reimpreso en HILPINEN, 1971: 36-58.

KRAWIETZ, W. et al. (eds.), 2000: The Reasonable as Rational? On Legal Argumentation and Justification. Festschrift for Aulis Aarnio, Berlin: Duncker \& Humblot.

KRIPKE, S., 1963: «Semantical Analysis of Modal Logic I: Normal Propositional Calculi», Zeitschrift für Mathematische Logik und Grundlagen der Mathematik, 9: 67-96.

LEWIS, D., 1986: On the Plurality of Worlds, Oxford: Blackwell.

Makinson, D., 1999: «On a Fundamental Problem of Deontic Logic», en McNamara y PRAKKEN, 1999: 29-53.

MAKINSON, D., y L. VAN DER TORRE, 2000: «Input-output Logics», Journal of Philosophical Logic, 29: 383-408.

- 2001: «Constraints for Input-output Logics», Journal of Philosophical Logic, 30 (2): 155185.

- 2003: «Permission from an Input-output Perspective», Journal of Philosophical Logic, 32: 391-416.

McNamara, P., y H. Prakken (eds.), 1999: Norms, Logics and Information Systems, Amsterdam-Berlin-Oxford-Tokyo-Washington, DC: IOS Press.

Meyer, J. J. Ch., y R. Wieringa (eds.), 1993: Deontic Logic in Computer Science: Normative System Specification, Chichester-New York-Brisbane-Toronto-Singapore: Wiley \& Sons.

Montague, R., 1960: «Logical Necessity, Physical Necessity, Ethics, and Quantifiers», Inquiry: An Interdisciplinary Journal of Philosphy, vol. 3: 259-269.

PRIOR, A. N., 1954: «The Paradoxes of Derived Obligation», Mind, 63: 64-65.

Rodríguez, J. L., 2002: Lógica de los sistemas jurídicos, Madrid: Centro de Estudios Políticos y Constitucionales.

- 2003: «Naturaleza y lógica de las proposiciones normativas. Contribución en homenaje a G. H. von Wright», Doxa, 26: 87-108.

Rönnedal, D., 2010: An Introduction to Deontic Logic, Stockholm: CreateSpace.

Ross, A., 1941: «Imperatives and Logic», Theoria, 7: 53-71.

Stalnaker, R. C., 2003: Ways a World Might Be. Metaphysical and Antimetaphysical Essays, Oxford: Clarendon Press.

Von Wright, G. H., 1951: «Deontic Logic», Mind, 60: 1-15.

- 1963a: Norm and Action. A Logical Inquiry, London: Rouledge \& Keagan Paul.

- 1963b: The Logic of Preference, Edinburgh: Edinburgh University Press.

- 1983: Practical Reason. Philosophical Papers, vol. I, Oxford: Basil Blackwell. 
— 2000: «On norms and Norm-Propositions. A Sketch», en KRAWIETZ, 2000: 173-178.

WoleńsKI, J., 1990: «Deontic Logic and Possible Worlds Semantics: A Historical Sketch», Studia Logica, 49, núm. 2: 273-282.

ZuletA, H., 2008: Normas y Justificación. Una investigación lógica, Madrid-Barcelona-Buenos Aires: Marcial Pons. 